\title{
The Effect of Material Composition of Piezoelectric Elements with Chosen Shapes on Plate Vibration Reduction
}

\author{
J. WICIAK AND R. TROJANOWSKI* \\ Department of Mechanics and Vibroacoustics, AGH University of Science and Technology, Al. A. Mickiewicza 30, \\ 30-059 Krakow, Poland
}

\begin{abstract}
This paper presents numerical analysis of efficiency of different shapes and material composition of piezoelectric actuators used for vibration reduction. Four numerical models representing a plate clamped on all sides with two attached piezoelectric elements were created. For each model, one element had the same shape and composition and was used for plate excitation, and the other one, used for vibration reduction, had different shape and possibly material composition and could be of square or circular shape with either homogeneous or two-part material composition. Two modes were taken into account: the first mode and the fifth mode. Analysis of results was performed with ANSYS software.
\end{abstract}

DOI: 10.12693/APhysPolA.125.A-179

PACS: 43.40.-r, 43.28.Js, 46.40.Ff

\section{Introduction}

For more than 20 years, piezoelectric elements have been used for active methods. Starting with works of Dimitriadis, Fuller, and Rogers [1], these materials are typically used for beams [2-4] and plate structures [5$10]$. Using piezo elements in analytical $[2,5,9,10]$ and numerical models $[3,8,10]$ or physical experiments $[6,7]$ shows the possibility to use them for significant vibration or noise reduction. These actuators are usually homogeneous square-shaped actuators. There are very few works concerning different compositions of piezo actuators, e.g. [3]. These works usually relate to functionally graded materials (FGM), where material properties change with thickness depending on the volume fraction of constituent materials $[11,12]$. This paper concentrates on using two-part elements with a step change in composition. Elements of that kind could be manufactured without too much difficulty.

\section{Description of analysis}

The subject of this paper is to determine whether a two-part piezo actuator can be an improvement over the standard homogeneous actuator. For this purpose, four numerical models consisting of a steel plate $(400 \mathrm{~mm} \times$ $400 \mathrm{~mm} \times 2 \mathrm{~mm}$ ) with attached piezo elements (Fig. 1) were created using ANSYS software.

For each model, one of the piezo elements was always a square based homogeneous element and was used for plate excitation. The other element, used for vibration reduction, had different shape or composition (Fig. 2). The considered shapes and composition were:

*corresponding author; e-mail: roman.cz.trojanowski@agh.edu.pl

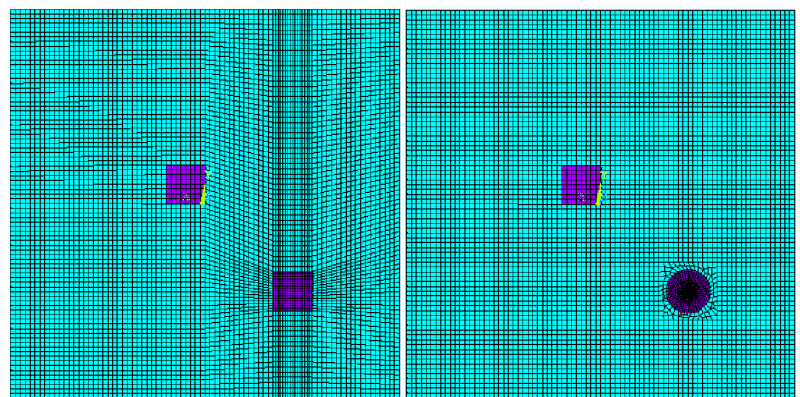

Fig. 1. Created models.

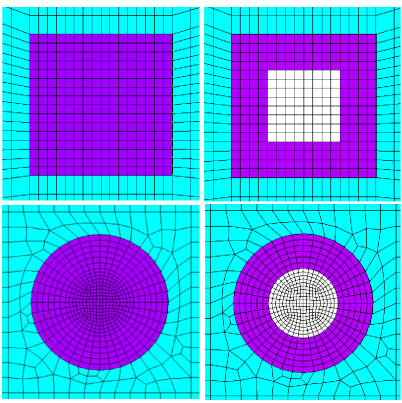

Fig. 2. The modelled piezo actuators: left column homogeneous, right column - two-part.

- square based homogeneous element with $a=$ $40 \mathrm{~mm}$ and $h=1 \mathrm{~mm}$;

- circle based homogeneous element with $r=$ $40 / \pi \mathrm{mm}$ and $h=1 \mathrm{~mm}$

- square based 2-part element with $a=40 \mathrm{~mm}$ and $h=1 \mathrm{~mm}$ (the inner part area is $1 / 4$ of the whole element area);

- circle based 2-part element $r=40 / \pi \mathrm{mm}$ and $h=1 \mathrm{~mm}$ (the inner part area is $1 / 4$ of the whole element area). 
Therefore all actuators had the same base area $A=$ $1600 \mathrm{~mm}^{2}$ which, in case of two-part elements, was divided between the inner part and the outer part where the inner part constituted $1 / 4$ of the whole element area.

Elements used for modeling the plate and the piezo elements and material properties were as follows:

- Plate: SOLSH190 $-E=1.93 \times 10^{11} \mathrm{~Pa}$, $\nu=0.29, \rho=7800 \mathrm{~kg} / \mathrm{m}^{3}$;

- Piezo element plates used for excitation: SOLID226 with PZ 28;

- Piezo element plates used for actuators: SOLID226 with PZ 28 and PZ 29;

- Properties of PZ 28: $\rho=7.70 \times 10^{3} \mathrm{~kg} / \mathrm{m}^{3}$, $d_{31}=-1.14 \times 10^{-10} \mathrm{~m} / \mathrm{V}, d_{33}=2.75 \times 10^{-10} \mathrm{~m} / \mathrm{V}$, $d_{15}=4.03 \times 10^{-10} \mathrm{~m} / \mathrm{V}, s_{11}^{\mathrm{E}}=1.26 \times 10^{-11} \mathrm{~Pa}^{-1}$, $s_{33}^{\mathrm{E}}=1.83 \times 10^{-11} \mathrm{~Pa}^{-1}, s_{12}^{\mathrm{E}}=-3.71 \times 10^{-12} \mathrm{~Pa}^{-1}$, $s_{13}^{\mathrm{E}}=6.60 \times 10^{-12} \mathrm{~Pa}^{-1}, s_{44}^{\mathrm{E}}=3.77 \times 10^{-11} \mathrm{~Pa}^{-1}$, $s_{66}^{\mathrm{E}}=3.26 \times 10^{-11} \mathrm{~Pa}^{-1}, \frac{\varepsilon_{11}^{S}}{\varepsilon_{0}}=1.22 \times 10^{3}$, $\frac{\varepsilon_{33}^{S}}{\varepsilon_{0}}=9.90 \times 10^{2}$;

- Properties of PZ 29: $\rho=7.46 \times 10^{3} \mathrm{~kg} / \mathrm{m}^{3}$, $d_{31}=-2.43 \times 10^{-10} \mathrm{~m} / \mathrm{V}, d_{33}=5.74 \times 10^{-10} \mathrm{~m} / \mathrm{V}$, $d_{15}=7.24 \times 10^{-10} \mathrm{~m} / \mathrm{V}, s_{11}^{\mathrm{E}}=1.70 \times 10^{-11} \mathrm{~Pa}^{-1}$, $s_{33}^{\mathrm{E}}=2.29 \times 10^{-11} \mathrm{~Pa}^{-1}, s_{12}^{\mathrm{E}}=-5.78 \times 10^{-12} \mathrm{~Pa}^{-1}$, $s_{13}^{\mathrm{E}}=8.79 \times 10^{-12} \mathrm{~Pa}^{-1}, s_{44}^{\mathrm{E}}=3.80 \times 10^{-11} \mathrm{~Pa}^{-1}$, $s_{66}^{\mathrm{E}}=4.56 \times 10^{-11} \mathrm{~Pa}^{-1}, \frac{\varepsilon_{11}^{S}}{\varepsilon_{0}}=2.44 \times 10^{3}$, $\frac{\varepsilon_{33}^{S}}{\varepsilon_{0}}=2.87 \times 10^{3}$.

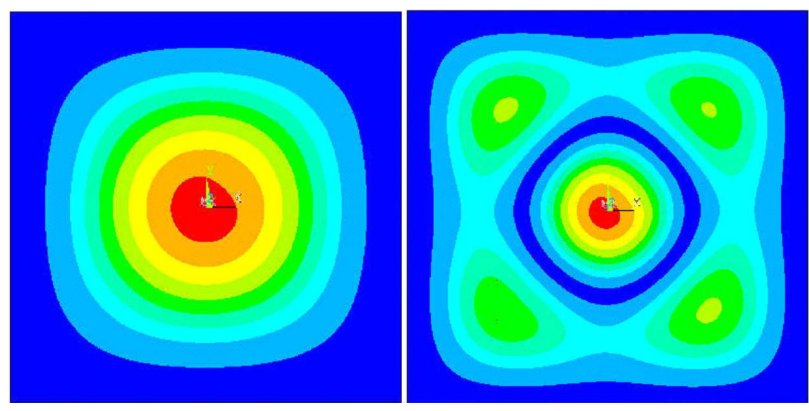

Fig. 3. Mode shapes selected for the analyses.

First, modal analysis was performed for the first six mode shapes. For further analyses, first and fifth modes were chosen for harmonic analyses (Fig. 3). The amplitude of voltage applied to the element used for plate excitation was $V_{\mathrm{e}}=100 \mathrm{~V}$. Reduction of plate vibrations was obtained using internal ANSYS optimization procedures. The goal function was assumed in the form

$$
|\boldsymbol{J}|=\min \sum_{i=1}^{n} \boldsymbol{X}(i)_{\text {sum }},
$$

where: $\boldsymbol{X}(i)_{\text {sum }}$ - displacement vector sum in $i$-th node; $n$ number of nodes (in this case all nodes making up the base of the plate).
The design variables for the procedures were: $V_{\mathrm{a}}-$ amplitude and $\phi_{\mathrm{a}}$ - phase of voltage applied to the actuator. The number of procedure steps was 30 . After each completion of optimization procedure, the procedure was repeated with the ranges of amplitude and phase of the voltage being narrowed, with the final ranges being $5 \mathrm{~V}$ for the amplitude and $2^{\circ}$ for the phase angle.

\section{Results}

Tables I and II present result of performed simulations. The first column in each table describes the material used for piezo actuator and in case of two-part actuators, the first name refers to the inner layer, and the second one to the outer layer. The second column relates to the mode shape. In the third column, amplitude of the voltage applied to the actuator is given The next column is the phase angle of said voltage. The last but one column is the reduction obtained in the analysis. Finally, the last column is the voltage efficiency of the element in question. This parameter was obtained as a simplified way to determine the energy efficiency of different shape and composition of the modelled actuators. Based on the fact that each element has that same base area $A=1600 \mathrm{~mm}^{2}$, the parameter can be defined as

$$
\frac{V}{L}=\frac{L_{\mathrm{red}} V_{\mathrm{e}}}{V_{\mathrm{a}}},
$$

where: $L_{\mathrm{red}}$ - obtained vibration reduction; $V_{\mathrm{e}}-$ voltage (amplitude) applied to the piezo element used for plates excitation; $V_{\mathrm{a}}$ - voltage (amplitude) applied to the piezo actuator.

TABLE I

Results for the square based piezo actuators: material material used for inner/outer part of actuator; mode mode shape; $V_{\mathrm{a}}$ - amplitude of voltage applied to the element; $\phi_{\mathrm{a}}-$ phase angle of voltage applied to the element; $L_{\text {red }}$ - vibration reduction level obtained from the simulation; $V / L$ - voltage efficiency of the element.

\begin{tabular}{l|c|r|c|c|r}
\hline \hline $\begin{array}{l}\text { material } \\
\text { (in./out.) }\end{array}$ & mode & $V_{\mathrm{a}}[\mathrm{V}]$ & $\phi_{\mathrm{a}}\left[^{\circ}\right]$ & $\begin{array}{c}L_{\text {red }} \\
{[\mathrm{dB}]}\end{array}$ & $V / L$ \\
\hline PZ 28 & 1 & 366.42 & 180.03 & 21.48 & 5.86 \\
PZ 29/PZ 28 & 1 & 295.38 & 180.03 & 21.76 & 7.37 \\
PZ 29 & 1 & 198.33 & 180.00 & 21.28 & 10.73 \\
PZ 28/PZ 29 & 1 & 228.64 & 180.18 & 21.38 & 9.35 \\
PZ 28 & 5 & 159.65 & 360.00 & 19.36 & 12.13 \\
PZ 29/PZ 28 & 5 & 128.69 & 360.00 & 19.26 & 14.96 \\
PZ 29 & 5 & 86.78 & 360.00 & 19.46 & 22.43 \\
PZ 28/PZ 29 & 5 & 100.50 & 360.16 & 19.02 & 18.93 \\
& & & & &
\end{tabular}

From the results obtained for different composition of square based elements it can be seen that there are no significant differences between the homogeneous and 2part elements (Fig. 4). For both modes, the difference in obtained reduction is less than $0.5 \mathrm{~dB}$.

The same can be said about the circle based elements (Fig.5), although here the difference is even smaller (less 
TABLE II

As Table I for circle based piezo actuators.

\begin{tabular}{l|c|c|c|c|c}
\hline \hline $\begin{array}{l}\text { material } \\
\text { (in./out.) }\end{array}$ & mode & $V_{\mathrm{a}}[\mathrm{V}]$ & $\phi_{\mathrm{a}}\left[^{\circ}\right]$ & $\begin{array}{c}L_{\mathrm{red}} \\
{[\mathrm{dB}]}\end{array}$ & $V / L$ \\
\hline PZ 28 & 1 & 385.21 & 180.00 & 20.68 & 5.37 \\
PZ 29/PZ 28 & 1 & 308.91 & 180.04 & 20.65 & 6.68 \\
PZ 29 & 1 & 208.02 & 180.01 & 20.56 & 9.88 \\
PZ 28/PZ 29 & 1 & 240.90 & 180.03 & 20.68 & 8.59 \\
PZ 28 & 5 & 167.86 & 360.00 & 19.36 & 11.53 \\
PZ 29/PZ 28 & 5 & 134.61 & 360.01 & 19.22 & 14.28 \\
PZ 29 & 5 & Ta90.91 & 360.01 & 19.14 & 21.06 \\
PZ 28/PZ 29 & 5 & 105.53 & 360.01 & 19.35 & 18.34
\end{tabular}

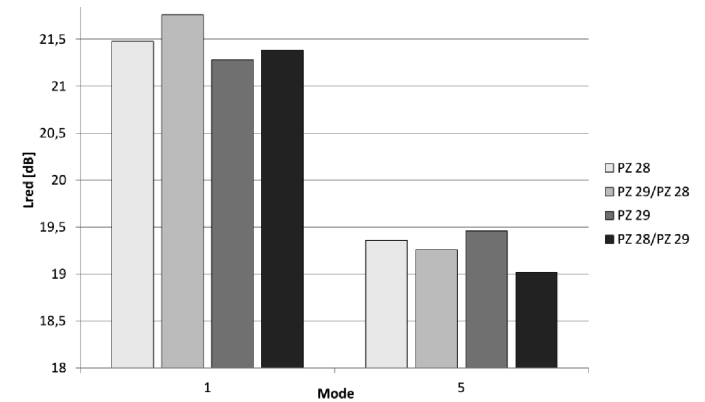

Fig. 4. Reduction obtained for square based piezo actuators.

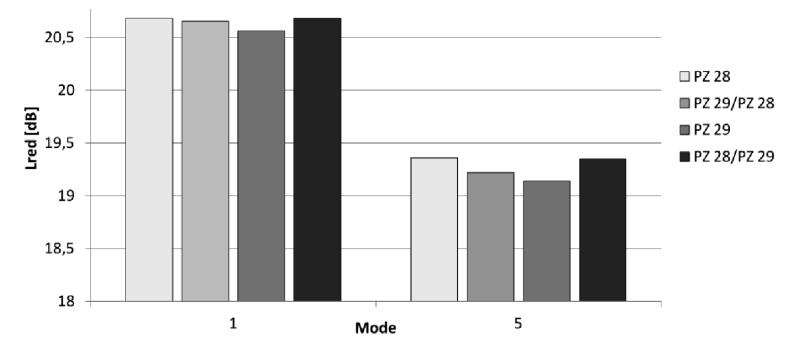

Fig. 5. Reduction obtained for circle based piezo actuators.

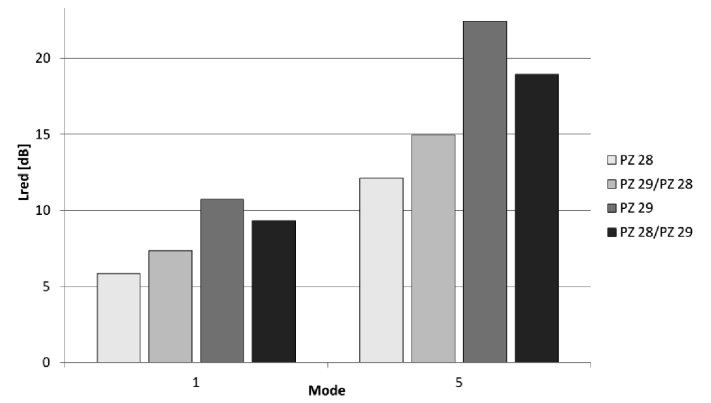

Fig. 6. Voltage efficiency of square based piezo elements.

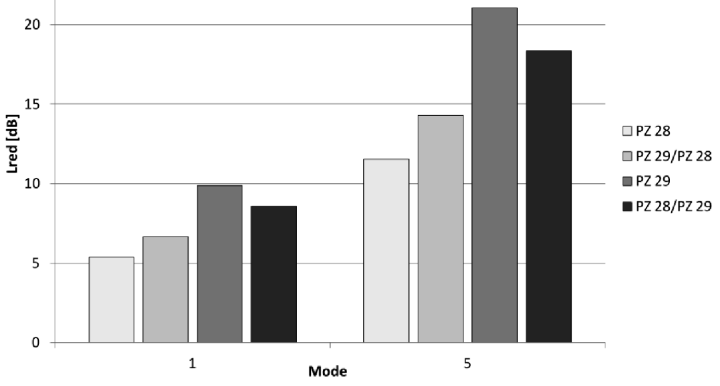

Fig. 7. Voltage efficiency of circle based piezo elements.

than $0.25 \mathrm{~dB})$. So it appears that a step change in composition does not improve it's effectiveness.

Still there are some changes in elements efficiency when different shapes are concerned (up to $1.1 \mathrm{~dB}$ ) with square based elements obtaining slightly higher vibration reduction.

When we look at the voltage efficiency of the modelled elements (Figs. 6 and 7) we can see that it increases proportionally to the substitute piezo electric constants of the element. This means that the greater is the share of the "stronger" material, the bigger is the voltage efficiency. Again, square based elements show slightly higher voltage efficiency compared to circle based elements.

\section{Conclusions}

This paper presents numerical analysis of the effects that different material compositions of piezo actuators with chosen shapes have on their efficiency when used as plate vibration reducing elements.

A reduction exceeding $21 \mathrm{~dB}$ for the 1st mode and $19 \mathrm{~dB}$ for the 5th mode in the case of square based elements was achieved. For circle based elements, the obtained reduction was more than $20,5 \mathrm{~dB}$ for the 1 st mode and more than $19 \mathrm{~dB}$ for the 5 th mode.

The performed simulations showed that a step change in composition of piezo elements did not influence their efficiency in a significant way. Vibration reduction levels obtained were roughly the same, and the differences between different compositions can be attributed to the optimization procedure.

For different material composition of the same shape of piezo actuator, the introduced voltage efficiency parameter changes proportionally to the substitute piezo electric constants. Out of the 2 shapes that were modelled, the rectangular elements seem to be more energy efficient.

It could be worth testing if changing the placement of the "inner" layer will result in any changes in the actuator's behavior.

It can also be theorized that with electrical separation of their inner and outer parts, these elements could be used as a sensor-actuator combo, with the inner layer being a sensor and the outer layer acting as an actuator. 
The efficiency of reduction should not be significantly impaired (especially when taking into account that the inner layer can be made smaller).

\section{Acknowledgments}

This study is a part of the research project $\mathrm{N}$ N504078038 supported by the Ministry of Science and Higher Education, Poland.

\section{References}

[1] E.K. Dimitriadis, C.R. Fuller, C.A. Rogers, J. Vib. Acoust. 113, 100 (1991).

[2] A. Brański, Acta Phys. Pol. A 123, 1123 (2013).

[3] U. Ferdek, M.S. Kozień, Acta Phys. Pol. A 123, 1044 (2011).
[4] M.S. Kozień, Acta Phys. Pol. A 123, 1029 (2013).

[5] E.M. Sekouri, Y. Hu, A.D. Ngo, Mechatronics 14, 1007 (2004)

[6] M. Kozupa, J. Wiciak, Acta Phys. Pol. A 118, 95 (2010).

[7] R. Trojanowski, J. Wiciak, Acta Phys. Pol. A 118, 168 (2010).

[8] A. Branski, S. Szela, Acta Phys. Pol. A 119, 942 (2011).

[9] M. Wiciak, Acta Phys. Pol. A 121, A-142 (2012).

[10] M. Wiciak, R. Trojanowski, Acta Phys. Pol. A 123, 1048 (2013).

[11] C.T. Loy, K.Y. Lam, J.N. Reddy, Int. J. Mech. Sci. 41, 309 (1999).

[12] M. Pietrzakowski, Mechanics 26, 181 (2007). 